\title{
Depression of Action Potential Characteristics and a Decreased Space Constant Are Present in Postischemic, Reperfused Myocardium
}

\author{
Joseph H. Levine, ${ }^{\star \ddagger}$ E. Neil Moore, ${ }^{\star}$ Harlan F. Weisman, ${ }^{\ddagger}$ Alan H. Kadish, ${ }^{\star}$ Lewis C. Becker, ${ }^{\ddagger}$ and Joseph F. Spear \\ *Department of Physiology, University of Pennsylvania School of Veterinary Medicine, Philadelphia, Pennsylvania 19104; and \\ ${ }^{\ddagger}$ Division of Cardiology, The Johns Hopkins University School of Medicine, Baltimore, Maryland 21205
}

\begin{abstract}
Brief periods of ischemia and reperfusion may lead to arrhythmias and delayed epicardial activation. To determine the nature of the electrophysiologic substrate and to gain insight into potential mechanisms underlying the electrophysiologic and hemodynamic abnormalities that develop in this setting, standard microelectrode techniques were used to measure action potential characteristics, conduction velocity, and space constants in canine isolated epicardial preparations removed after a 15-min anterior descending artery occlusion and 20 -min reflow period in vivo. Our results demonstrate a significant reduction in conduction velocity $(0.78 \pm 0.38$ vs. $0.31 \pm 0.12 \mathrm{~m} / \mathrm{s}, P<0.001)$, space constant (1.05 \pm 0.42 vs. $0.45 \pm 0.12 \mathrm{~mm}, P=0.004)$, resting membrane potential $(81.3 \pm 2.5$ vs. $61.7 \pm 7.8 \mathrm{mV}, P<0.001)$, action potential amplitude $(94.1 \pm 4.2$ vs. $64.1 \pm 1.5 \mathrm{mV}, P<0.001)$, and $\mathrm{d} V / \mathrm{d} T(164.7 \pm 37.3 \mathrm{vs} .52 .6 \pm 19.7 \mathrm{~V} / \mathrm{s}, P<0.001)$ in postischemic reperfused myocardium. The space constant and $\mathrm{d} V / \mathrm{d} T$ each correlated with conduction velocity; in addition, the space constant was an independent predictor of conduction velocity in these tissues. These electrophysiologic abnormalities may play a role in the arrhythmias and abnormalities of contraction present in postischemic, reperfused myocardium.
\end{abstract}

\section{Introduction}

Ventricular tachyarrhythmias may occur during reperfusion after coronary artery occlusion (1-7). The electrophysiologic basis for arrhythmias that occur immediately after reperfusion has been well described. Several groups have demonstrated delayed, fractionated electrograms in postischemic myocardium immediately after reperfusion (4-6). In addition, sudden changes in action potential characteristics and refractoriness have been documented during this time (8). The electrophysiologic mechanisms underlying later (5-30 min) reperfusion arrhythmias, on the other hand, are not as well understood. Whether automaticity or reentry underlies the genesis of these arrhythmias has been the subject of several studies with varying results $(3-5,9)$.

We have recently demonstrated that delayed epicardial activation can be identified in some areas of myocardium subjected to sublethal, ischemic injury in vivo (10). The cellular basis for these changes, however, is unknown. Alterations in ionic currents

Address correspondence to Dr. Levine, Johns Hopkins Hospital, Carnegie 592, 600 N. Wolfe Street, Baltimore, MD 21205.

Received for publication 28 April 1986 and in revised form 11 August 1986.

J. Clin. Invest.

(c) The American Society for Clinical Investigation, Inc.

0021-9738/87/01/0107/10 \$1.00

Volume 79, January 1987, 107-116 (membrane effects) as well as changes in the space constant might contribute to a delay in epicardial activation. We and other investigators have measured depressed action potential characteristics $(9,11-15)$ and decreased space constants (16-18) associated with acute hypoxia and chronic infarction. Whether these changes may also be present in myocardium after sublethal ischemic injury, however, is unknown.

The purpose of the present experiments was to document and characterize the impairment of conduction present in postischemic, reperfused myocardium, to determine whether a decreased space constant or depressed action potential characteristics could be demonstrated, and to determine the roles of changes in active membrane characteristics and space constants in the decrement of conduction measured after sublethal ischemic injury.

\section{Methods}

Electrophysiological methods. Experiments were performed on 32 tissues removed from 15 beagles weighing $10-20 \mathrm{~kg}$. The animals were anesthetized with intravenous sodium pentobarbital $(30 \mathrm{mg} / \mathrm{kg}$ body weight), and their chests were opened via a left lateral thoracotomy. The left anterior descending artery was occluded distal to the first diagonal branch for $15 \mathrm{~min}$ and then released. A 20-min recovery period followed and the hearts were then removed. One or two epicardial tissues 1-2 mm thick, $2 \mathrm{~cm}$ wide, and $2-3 \mathrm{~cm}$ long were removed from the ischemic zone (within $2 \mathrm{~cm}$ of the second diagonal branch) of 15 hearts so that their longitudinal axis was parallel to the superficial fiber orientation. One or two control tissues from the hearts of 10 of the animals were removed from the nonischemic circumflex region. The tissues were placed in a 100-ml tissue bath and superfused with standard Tyrode's solution (flow rate, $10 \mathrm{ml} / \mathrm{min}$ ). The tissues were paced at a basic cycle length of $1000 \mathrm{~ms}$ using bipolar stimulation electrodes consisting of two Tefloncoated silver wires and allowed to equilibrate for $30 \mathrm{~min}$. Constantcurrent rectangular wave pulses of $2 \mathrm{~ms}$ and twice diastolic threshold intensity were used. Our techniques have been described in detail previously (15). Using these techniques, thin epicardial tissues such as those used in these experiments can be maintained without a significant decrement in the electrophysiologic properties for up to $5 \mathrm{~h}$, which indicates that perfusion and oxygenation are adequate in the superficial cell layers studied.

With standard 3-M potassium-filled microelectrodes, transmembrane action potentials were recorded from cells in the superficial layers of the epicardial preparations. The action potentials, as well as an electronically differentiated signal that measured $\mathrm{d} V / \mathrm{d} T$, were displayed on an oscilloscope and photographed on $35-\mathrm{mm}$ film. Action potential parameters were measured to the nearest $0.1 \mathrm{mV}$ and $0.1 \mathrm{~ms}$ by projecting the 35 $\mathrm{mm}$ film onto a manual digitizer (GTCO Corp., Rockville, MD) interfaced with a computer (model 9836; Hewlett Packard Co., Sunnyvale, CA). The action potential parameters measured were resting potential, action potential amplitude, maximum rate of depolarization, and action potential duration at $100 \%$ repolarization. The action potential foot was digitized to $10 \mathrm{mV}$. The time constant was determined from a semilogarithmic plot of voltage vs. time. In five tissues from the ischemic zones, no active responses were noted during pacing at current strengths up to $5 \mathrm{~mA}$. These tissues did maintain resting potentials of $-50--60 \mathrm{mV}$ 
over a minimum 3-h observation period. Six to eight impalements were made but we did not fully map these tissues to prove that no active propagated responses were present. Similarly, because changes in pulse strength and duration may affect action potential characteristics, we did not feel justified in presenting data during stimulation under conditions of marked increases in these parameters. Finally, we did not determine a change in pacing site might have affected the ability to generate propagated responses. Thus, our summary data as well as cable analysis reflect the results of only those tissues with propagated responses.

Conduction velocity was determined from multiple microelectrode recordings made parallel to the fiber orientation. Starting $\sim 5 \mathrm{~mm}$ from the stimulating electrode, sequential transmembrane potential recordings were made approximately every $0.5 \mathrm{~mm}$ over a total of 4-10 $\mathrm{mm}$. Distances between recording sites were measured using a dissecting microscope with an optical micrometer. The site of cell impalement was determined by observing the midpoint of the dimple produced by the microelectrode impaling the surface of the tissue (resolution $0.08 \mathrm{~mm}$ ). The activation time was defined as the time difference between the stimulus artifact of a single impalement and the time of maximum rate of depolarization of a second action potential recorded using a roving microelectrode parallel to fiber orientation. Conduction velocity was determined from a plot of activation time vs. distance using the slope of the linear regression. These methods provide an average conduction velocity between the multiple sites. Because the slope is used, the value obtained is independent of stimulus latency. An $r^{2}$ value of 0.90 for the regression was considered acceptable and indicated linear conduction over at least $5 \mathrm{~mm}$ in the area of interest $(15,17)$. In these tissues, an extracellular, unipolar electrogram was also recorded to confirm uniform, longitudinal conduction on a cell to cell basis (19). For tissues exhibiting nonuniform conduction $\left(r^{2}<0.90\right.$ or notches in the extracellular electrogram), the slope of the regression provides only an approximation for overall conduction velocity in the tissues and hence the technique is limited under these conditions. The latter conditions were noted in six tissues and as such the values were not included in the analytical relationships used to test this model as these relationships are based upon the assumption that uniform conduction is present.

Space constants were determined using a technique previously described $(16,17)$. A silver-silver chloride-tipped extracellular contact electrode was positioned at one end of the tissue such that a monophasic action potential was recorded, indicating partial electrical access to the intracellular space. Subthreshold rectangular depolarizing current pulses (10-30 $\mu \mathrm{A}, 80$-ms duration), delivered by a constant current source, were passed between the silver-silver chloride electrode at the catheter tip and a bath ground. 4-10 sequential transmembrane potentials were sampled at distances up to $2 \mathrm{~mm}$ from the wall of the contact electrode using a 5-7,000 differential electrometer (World Precision Instruments, New Haven, CT) that measured the difference between the roving microelectrode and a reference microelectrode positioned in the bath within $0.5 \mathrm{~mm}$ of the recording microelectrode. During the space-constant determinations the tissue was paced at a basic cycle length of $1,000 \mathrm{~ms}$ and the current pulse was delivered after the cell had undergone repolarization following a conducted beat. The electrotonic potentials were displayed on an oscilloscope and photographed on $35-\mathrm{mm}$ film. The amplitude of the deflection was digitized to the nearest $0.1 \mathrm{mV}$ and the distance of the impalement from the contact electrode determined as described above. The space constant was determined from a semilogarithmic plot of the steady-state electrotonic potential vs. distance, assuming an infinite linear cable, and was defined as the distance at which the potential at the site of injection $\left(V_{0}\right)$ fell to a value of $V_{0} / e$. Spaceconstant determinations were performed in 18 (8 control and 10 postischemic) tissues. Space-constant determinations were not performed in the other tissues due primarily to two factors: $(a)$ in 7 tissues (two control and five postischemic), even very low current strength led to regenerative activation of the tissue; and $(b)$ space-constant determinations were not attempted in two tissues (one control and one postischemic) with active responses and in all five tissues without active response.

As in all multicellular preparations in which cable analysis has been applied, certain assumptions must be made and the analysis can provide only an approximation. When measuring space constants in cardiac muscle, a method that can deliver current to a large number of cells simultaneously is of import. The theoretical basis and justification for this method of space-constant determination have been described and are based upon the normal anisotropy of ventricular muscle $(16,17,19$ 21). Clerc (21) found a 9:1 difference in the internal resistance between the transverse and longitudinal directions. He and others $(19,22)$ have attributed the anisotropy to a relative decrease in low resistance connections in the direction perpendicular to fiber orientation. Similarly, conduction from the superficial to deep fibers in our tissues was transverse to fiber orientation. In the present experiments, the large diameter of the contact electrode relative to fiber dimension assured a uniform distribution of intracellular current among a large population of cells, and the anisotropic nature of the preparation offered a preferential path for current flow in one spatial dimension. Because all space-constant determinations in the present experiments were performed parallel to the fiber orientation, the preferential path resulting from anisotropy may have accounted for the exponential fall-off of voltage with distance, and provides some basis for using the assumption of a one-dimensional cable in calculating the space constant. The finding that the space-constant determinations are little influenced by the absolute amount of subthreshold current injected using this technique (17) is further support for the validity of the method. Similarly, the results from previous (17) as well as the current experiments were tested using the analytical relationships expected from cable analysis between conduction velocity, the space constant, the action potential foot, and action potential characteristics. That these data fit predicted relationships in a one-dimensional cable model further suggests that this methodology is justified in approximating determinants of conduction. A drawback to this method of determining the space constant is that the exact ratio of intracellular to extracellular current cannot be measured. The input resistance can therefore not be determined and cannot be used to determine membrane resistance. Another problem attending the use of depolarizing responses to quantitate the space constant is that voltage-dependent active components, rectification of steady-state outward currents and voltage-dependent steady-state inward currents may contribute to the subthreshold response. These factors will tend to cause a deviation from a purely exponential voltage drop with distance of the subthreshold response. Unfortunately, hyperpolarizing pulses are frequently accompanied by regenerative activation of the tissue and hence are not optimal to evaluate passive membrane properties either. Thus, our measurements represent an approximation of the true space constant. That the correlation coefficients of the regression of the exponential voltage drop with distance are $>0.9$ suggest that the error introduced by these factors is small.

Histological methods. Immediately after excision of the beating heart, specimens for light and electron microscopy were obtained from fresh tissue adjacent to the epicardial myocardium removed for the electrophysiological study. These specimens were $\sim 1-2-\mathrm{mm}$ thick and were cut into $1-\mathrm{mm}^{3}$ blocks. In addition, specimens were obtained in a similar fashion from two tissues that were maintained in the tissue bath for 3 and $5 \mathrm{~h}$. The tissue samples were then immediately fixed in cold $3 \%$ glutaraldehyde in $0.1 \mathrm{M}$ phosphate buffer for $24 \mathrm{~h}$. After three 10-min washes with cold $0.1 \mathrm{M}$ phosphate buffer, the tissue was postfixed with cold $1 \%$ osmium tetroxide for $1 \mathrm{~h}$, followed by three additional 5 -min washes in cold distilled water. The tissue was dehydrated in a graded series of alcohols and xylene and then incubated in a 50:50\% mixture of xylene and epoxy resin at $60^{\circ} \mathrm{C}$. After $1 \mathrm{~h}$, the tissue was embedded in pure epoxy resin and kept at $60^{\circ} \mathrm{C}$ for $12 \mathrm{~h}$. Semi-thin $(1-\mu \mathrm{m})$ sections of the epoxy resin-embedded tissue were stained with toluidine blue and examined with a light microscope. Ultrathin (100-nm) sections were prepared from those tissue blocks with fibers oriented longitudinally (four to six blocks from each specimen). The ultra-thin sections were mounted on copper grids and stained with lead citrate and uranyl acetate. Two grids of serial sections were made from each of the selected blocks and one of the grids was randomly chosen for electron microscopy (100S electron microscope; Jeol USA, Inc., Peabody, MA). The entire grid was scanned and representative photographs obtained. Sections of tissue blocks cut perpendicular to the epicardial surface with fibers arranged 
in cross-section were selected for cell cross-sectional areas and diameter determinations. Cell measurements were made using a light microscope interfaced with a Zeiss Videoplan microcomputer at a magnification of 500. Cross-sectional myocyte profiles containing nuclei were traced and digitized using a cursor projected into the microscope field via a camera lucida. A minimum of 50 myocyte profiles per specimen were measured.

Statistical methods. Parameter means were compared using an analysis of variance. Differences between groups were tested at the 0.05 level using the Newman-Keuls multiple rank test. All tabular data is expressed as means and standard deviations. Linear fits were made using linear regression techniques. Exponential fits were made by linear regression of the semilogarithmic transform of the data. A $P \leq 0.05$ was considered significant for these determinations.

\section{Results}

\section{Action potential characteristics after brief ischemia and reperfusion}

In Fig. 1 are examples of analog recordings of action potentials from postischemic, reperfused myocardium. All four action potentials demonstrate a reduction in resting potential, action potential amplitude, maximum rate of depolarization, and action potential duration. As in the examples shown, these changes were quite variable and ranged from relatively minor abnormalities to the appearance of only subthreshold depolarizations. No normal action potentials were recorded in these tissues. Table I presents the mean values for these parameters recorded in the control preparations and in the 16 preparations that underwent brief ischemia and reperfusion in vivo that demonstrated active responses. A significant reduction in resting potential, action potential amplitude, maximum rate of depolarization, and action potential duration was present in each postischemic, reperfused tissue studied and the mean values for each parameter were significantly different from the control tissues.

A slow improvement in the action potential parameters with time was seen in a few of the tissues. Eight tissues were followed 2-5 h (mean, $3.4 \mathrm{~h}$ ). In five tissues, no improvement was noted. In the other three, a tendency toward slow improvement in action potential parameters with time was noted. Mean resting potential increased from 52.0 to $65.7 \mathrm{mV}$, action potential amplitude from 12.0 to $34.0 \mathrm{mV}$, and $\mathrm{d} V / \mathrm{d} T$ from 6.6 to $48.4 \mathrm{~V} / \mathrm{s}$. In one tissue followed $5 \mathrm{~h}$, depressed action potential parameters persisted but approached control values over time.

Conduction velocity was determined as the slope of the
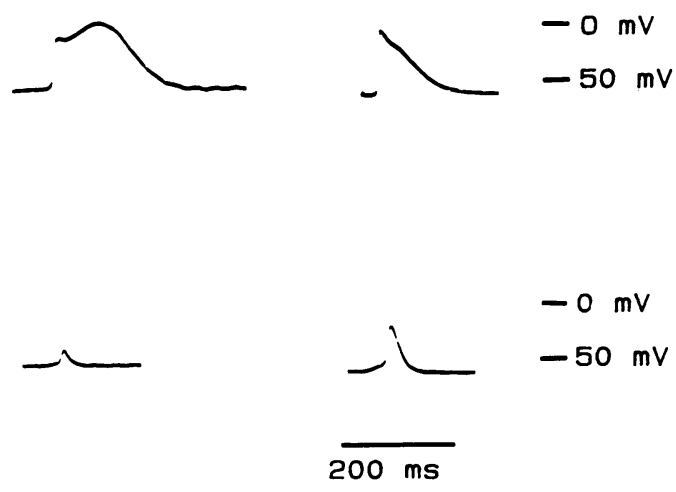

Figure 1. Examples of action potentials from postischemic, reperfused myocardium. Each action potential shown is abnormal. A decrease in resting potential, action potential amplitude, and the maximum rate of depolarization were seen in each tissue. In some cases (bottom left), only subthreshold depolarizations were present. best-fit linear regression of activation time versus distance from the stimulating electrode. Fig. 2 shows data from a representative control normal tissue and a postischemic, reperfused tissue. Note that the relationship between activation time and distance was linear in both cases $\left(r^{2}=0.99\right)$ but that the conduction velocity was slower in the postischemic, reperfused tissue. The measured conduction velocities for each tissue are shown in Table I.

Conduction was linear in each control tissue with a mean conduction velocity of $0.78 \pm 0.11 \mathrm{~m} / \mathrm{s}$. In the postischemic, reperfused tissues, on the other hand, two different kinds of slow conduction were identified. In the first type, conduction appeared uniformly slow; that is, as in Fig. 2, there was a good linear fit of the data $\left(r^{2}>0.90\right)$. In regions with this type of conduction, conduction velocity ranged between 0.16 and $0.52 \mathrm{~m} / \mathrm{s}$ with a mean of $0.31 \pm 0.04 \mathrm{~m} / \mathrm{s}$. The second type of slow conduction was nonuniform; large increases in activation times occurred between closely adjacent cells in some portions of the tissue while conduction was more rapid in others. It was in these tissues that subthreshold responses and prepotentials were most commonly seen. These phenomena have been previously described in the setting of nonuniform conduction and have been atributed to discontinuous propagation between cells $(15,17)$. The approximate conduction velocity in the tissues that exhibited slow nonuniform conduction ranged between 0.07 and $0.47 \mathrm{~m} / \mathrm{s}$; the mean value of $0.25 \pm 0.05 \mathrm{~m} / \mathrm{s}$ was significantly different from that recorded in the normal control tissues but did not differ significantly from that recorded in the postischemic, reperfused tissues that exhibited linear conduction.

\section{Space constant after brief ischemia and reperfusion}

Fig. 3 illustrates our method for determining the space constant. Shown are subthreshold electrotonic depolarizations in response to intracellular current injection after a conducted action potential. Recordings were made at the stated distances from the extracellular electrode through which the current was injected. The amplitude of the subthreshold depolarization decreased at increasing distances from the extracellular electrode. The relationship between the steady-state electrotonic potential amplitude and distance from the extracellular electrode was exponential. In this example from a control tissue, the regression line had an $r^{2}$ value of 0.98 and the measured space constant was $1.26 \mathrm{~mm}$. The simple exponential decay of the steady-state electrotonic potential with distance supports the use of this technique for measuring space constants. Fig. 4 presents semilogarithmic plots of the steady-state electrotonic potential amplitude vs. distance for typical control and postischemic, reperfused tissues. The regressions for the control and postischemic tissues had $r^{2}$ values of 0.95 and 0.99 , respectively. The fall-off of amplitude with distance was greater in the postischemic, reperfused tissue than the control tissue. The measured space constant in the control tissue was $0.73 \mathrm{~mm}$, compared with $0.44 \mathrm{~mm}$ in the postischemic, reperfused tissue. The mean correlation coefficients were $0.94 \pm 0.03$ and $0.95 \pm 0.05$ for control and postischemic, reperfused tissues, respectively. Table II shows the results of the space-constant determinations for 8 control and 10 postischemic, reperfused tissues. The mean space constant in the control tissues was $1.05 \pm 0.42 \mathrm{~mm}$, compared with $0.45 \pm 0.12 \mathrm{~mm}$ in the postischemic, reperfused tissues exhibiting linear conduction and $0.51 \pm 0.08 \mathrm{~mm}$ in the postischemic, reperfused tissues exhibiting discontinuous conduction. The values of the space constants measured in the postischemic, reperfused myocardium differed significantly from those measured in the normal control tissues. 
Table I. Action Potential Characteristics in Postischemic, Reperfused Myocardium

\begin{tabular}{|c|c|c|c|c|c|}
\hline Tissue & $\begin{array}{l}\text { Resting membrane } \\
\text { potential }\end{array}$ & $\begin{array}{l}\text { Action potential } \\
\text { amplitude }\end{array}$ & $\mathrm{d} V / \mathrm{d} T$ & $\begin{array}{l}\text { Action potential } \\
\text { duration }\end{array}$ & Conduction velocity \\
\hline & $m V$ & $m V$ & $V / s$ & $m s$ & $m / s$ \\
\hline \multicolumn{6}{|l|}{ Control } \\
\hline 1 & 77.6 & 91.1 & 125.2 & 211.7 & 0.56 \\
\hline 2 & 79.0 & 87.1 & 146.0 & 145.9 & 0.53 \\
\hline 3 & 79.4 & 98.2 & 154.8 & 164.6 & 0.45 \\
\hline 4 & 82.5 & 97.1 & 146.2 & 188.0 & 0.64 \\
\hline 5 & 81.2 & 92.0 & 134.3 & 132.1 & 0.81 \\
\hline 6 & 79.8 & 97.6 & 166.5 & 122.4 & 1.75 \\
\hline 7 & 84.0 & 90.4 & 198.0 & 158.0 & 1.22 \\
\hline 8 & 79.2 & 92.7 & 194.2 & 152.5 & 0.70 \\
\hline 9 & 83.5 & 99.8 & 160.6 & 190.9 & 0.56 \\
\hline 10 & 84.7 & 98.2 & 133.4 & 81.9 & 0.69 \\
\hline 11 & 83.8 & 90.6 & 252.4 & 142.0 & 0.63 \\
\hline Mean \pm SD & $81.3 \pm 2.5$ & $94.1 \pm 4.2$ & $164.7 \pm 37.3$ & $147.7 \pm 46.7$ & $0.78 \pm 0.38$ \\
\hline \multicolumn{6}{|c|}{ Postischemic (linear conduction) } \\
\hline 1 & 62.1 & 66.8 & 53.0 & 134.0 & 0.34 \\
\hline 2 & 63.9 & 76.8 & 44.3 & 100.9 & 0.22 \\
\hline 3 & 60.1 & 60.4 & 41.1 & 99.8 & 0.47 \\
\hline 4 & 60.6 & 55.4 & 47.4 & 85.1 & 0.30 \\
\hline 5 & 79.9 & 66.7 & 65.2 & 42.1 & 0.35 \\
\hline 6 & 62.7 & 31.0 & 38.0 & 74.9 & 0.26 \\
\hline 7 & 50.1 & 75.1 & 102.5 & 170.4 & 0.52 \\
\hline 8 & 65.2 & 61.7 & 47.3 & 100.5 & 0.23 \\
\hline 9 & 55.9 & 87.1 & 53.7 & 54.5 & 0.16 \\
\hline 10 & 56.0 & 60.0 & 33.3 & 71.8 & 0.22 \\
\hline Mean \pm SD & $61.7 \pm 7.8^{*}$ & $64.1 \pm 15.0^{*}$ & $52.6 \pm 19.7^{*}$ & $93.4 \pm 37.6^{*}$ & $0.31 \pm 0.12^{*}$ \\
\hline \multicolumn{6}{|c|}{ Postischemic (nonlinear conduction) } \\
\hline 1 & 58.7 & 67.5 & 86.0 & 51.8 & 0.47 \\
\hline 2 & 71.5 & 77.4 & 73.8 & 97.8 & 0.30 \\
\hline 3 & 55.4 & 11.6 & 2.0 & 19.3 & 0.29 \\
\hline 4 & 60.0 & 73.8 & 44.8 & 40.7 & 0.07 \\
\hline 5 & 50.0 & 32.9 & 23.6 & 19.5 & 0.14 \\
\hline 6 & 55.7 & 60.6 & 43.7 & 110.2 & 0.24 \\
\hline Mean \pm SD & $58.7 \pm 7.3^{*}$ & $54.0 \pm 26.1^{*}$ & $45.7 \pm 31.0^{*}$ & $56.6 \pm 39.0^{*}$ & $0.25 \pm 0.14^{*}$ \\
\hline $\begin{array}{l}P \text { (analysis o } \\
\quad \text { variance) }\end{array}$ & $<0.001$ & $<0.001$ & $<0,001$ & $<0.001$ & $<0.001$ \\
\hline
\end{tabular}

\section{Pathologic studies}

Electron microscopy. Seven specimens from the ischemic, reperfused zone were studied. Five of the seven specimens were entirely normal (Fig. $5 \mathrm{~A}$ ). In these, sarcomeres were in register exhibiting $A$ and $Z$ bands, but not $I$ bands. The sarcolemma composed of a glycocalyx and plasmalemma had a normal scalloped appearance. The sarcoplasm contained abundant granular glycogen. Nuclei were centrally located with even distribution of chromatin. Mitochondria had tightly packed cristae with a small volume of matrix space. The intercalated disks had the typical steplike configuration with transverse and longitudinal portions. The transverse portion contained mainly fascia adherentes and macula adherentes (desmosomes) (Fig. $5 A$, inset). The space between cells at the fascia adherentes was normal (250-300 A). Nexuses were more commonly seen along the lon- gitudinal portions of the intercalated disk and appeared as pentalaminar membrane interfaces between adjacent cells.

Two specimens were abnormal. In one, $30 \%$ of the fields viewed showed cells with mild mitochondrial swelling with some linearization of mitochondrial cristae, but no signs of irreversible injury were present. In the other, irreversible ischemic injury was apparent (Fig. $5 \mathrm{~B}$ ). In this tissue, a heterogeneous population of myocytes was present. Some cells showed only mild mitochondrial swelling and linearization of cristae. Other myocytes had moderate to marked swelling with increased sarcoplasmic space and marked decrease of glycogen granules. Nuclei showed margination of chromatin and mitochondria were swollen with disrupted cristae and increased matrix space. Amorphous matrix densities, a sign of irreversible ischemic injury, were present in some of the mitochondria. There was evidence of both irre- 


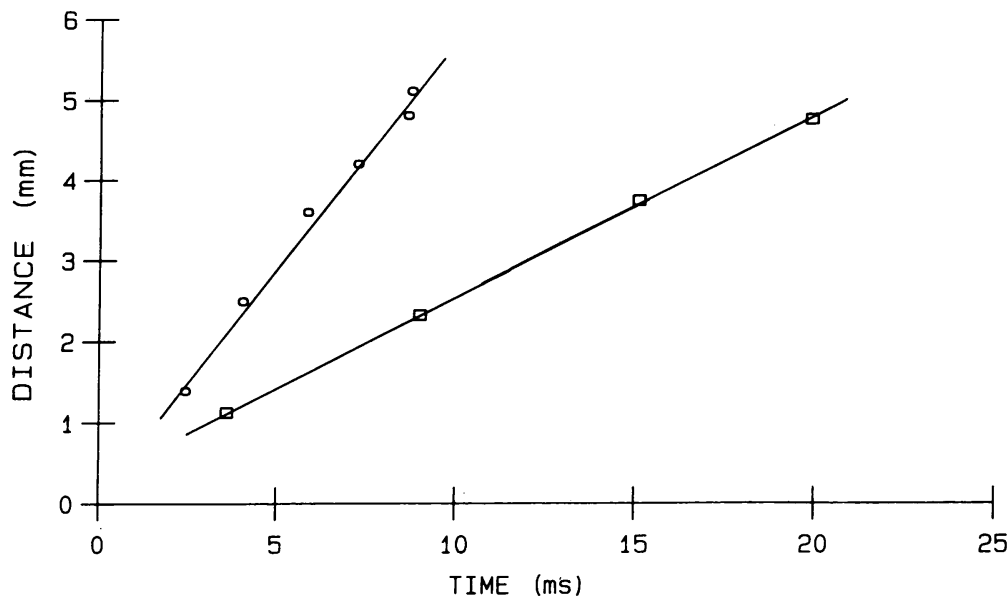

$0.4 \mathrm{~mm}$

$0.7 \mathrm{~mm}$

$1.4 \mathrm{~mm}$

2. $1 \mathrm{~mm}$

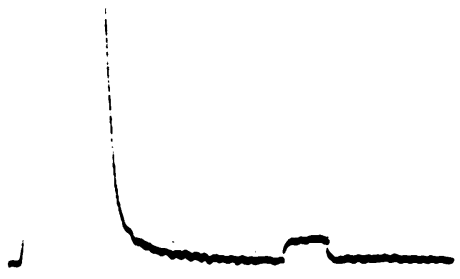

10

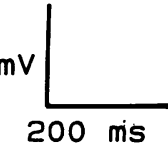

Figure 2. A plot of distance vs. activation time for normal (open circles) and postischemic, reperfused myocardium (open squares). Both plots were linear $\left(r^{2}=0.99\right)$, which indicated uniform conduction. The conduction velocity (the slope of the regression) was significantly reduced in the postischemic, reperfused tissues (from 0.56 to $0.22 \mathrm{~m} / \mathrm{s})$.

Figure 3. Analog records demonstrating the electrotonic responses measured at various distances from an extracellular electrode through which intracellular current was injected. Note that the amplitude of the steady-state voltage of the electronic potential decreased at increasing distances from the extracellular electrode. 


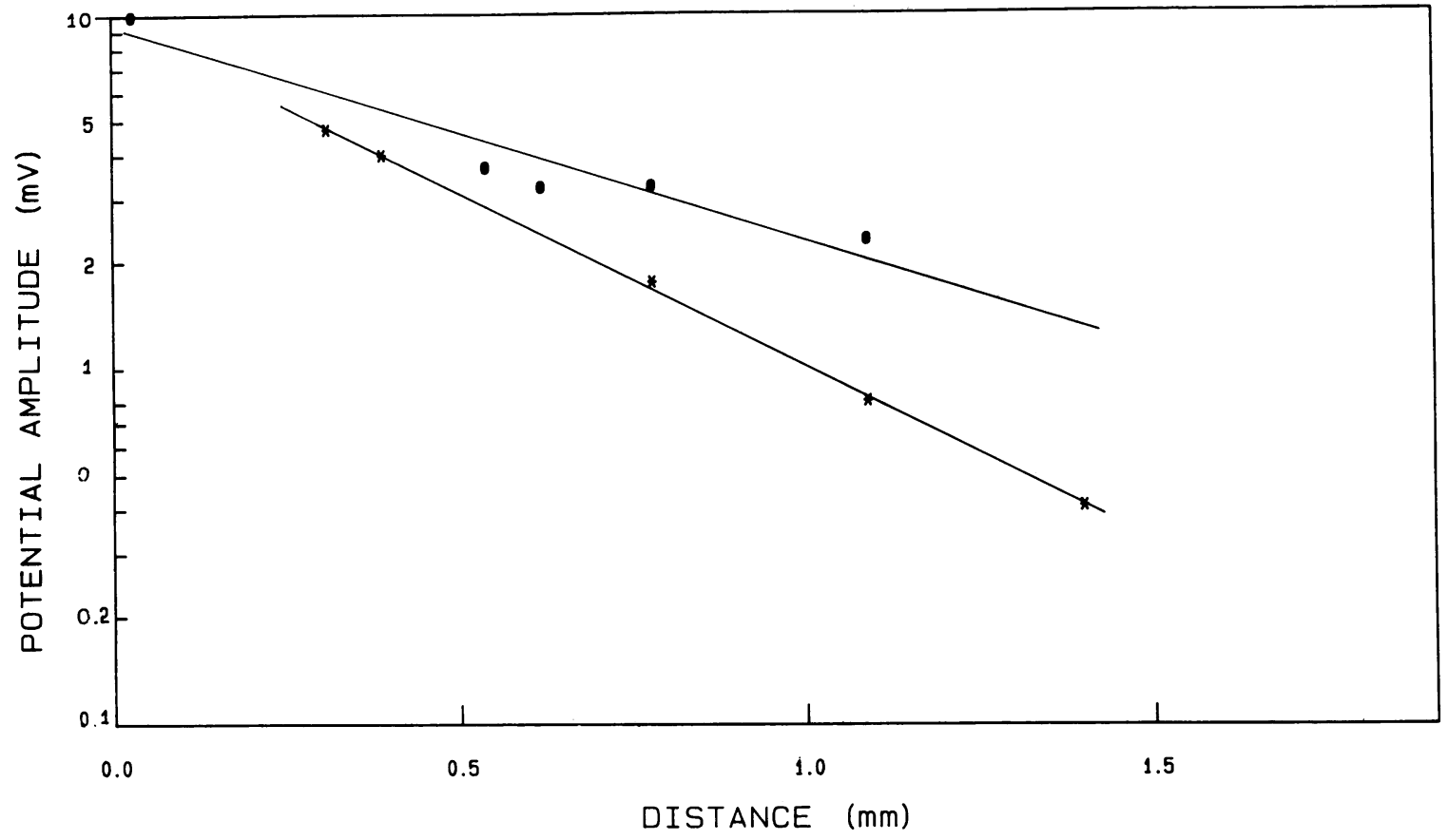

Figure 4. A semilogarithmic plot of steady-state potential amplitude vs. distance from the extracellular electrode current source. Shown are plots for a control (filled circles) and postischemic, reperfused tissue (asterik). Note that the fall-off of voltage with distances is greater in the postischemic, reperfused tissue and hence the measured space constant is lower $(0.44 \mathrm{~mm}$ compared with $0.73 \mathrm{~mm}$ for control).

versible ischemia and reperfusion injury: some sarcomeres were relaxed with prominent I bands; others were hypercontracted with contraction bands. In the relaxed region, intercalated disks appeared normal. In areas of hypercontraction, macula adherentes and facia adherentes were normal but the space between cells was increased (>300 A). No nexuses were seen.

Although the specimen that showed irreversible ischemic and reperfusion injury was one of the more severely affected tissues electrophysiologically (Table I, postischemic [uniform] tissue 3), there was no correlation between changes in action potential parameters or space constants and the histologic findings.

Cross-sectional fiber diameter. Mean cross-sectional area of the myocytes in the postischemic tissue was $242.4 \pm 60 \mu \mathrm{m}^{2}$. Assuming a circular cross-sectional shape, this area corresponds to a mean diameter of $17.6 \mu \mathrm{m}$, which is not significantly different from the myocyte diameter in normal tissue described previously (17).

\section{Discussion}

The present study demonstrates that brief periods of ischemia followed by reperfusion lead to significant cellular electrophysiologic abnormalities. The study documents impaired conduction, a decrease in the space constant, and a depression in each of the action potential parameters measured in the postischemic, reperfused tissues. In addition, our data demonstrate a strong direct relationship between the measured space constants and conduction velocity (Fig. 6) and a weaker but significant relationship between the square root of $\mathrm{d} V / \mathrm{d} T$ and conduction velocity. Thus, the impaired conduction measured in myocardium subjected to sublethal, ischemic injury may be due to alterations in both active membrane characteristics (ionic currents) and passive properties (intracellular resistance including nexuses, extracellular resistance, and cell size and shape).

A decrease in resting potential, action potential amplitude, maximal rate of depolarization, and action potential duration was present in each of the postischemic, reperfused tissues (Fig. 1, Table I). These findings in ventricular muscle, as well as a relatively prolonged time course of recovery are qualitatively similar to those seen in Purkinje fibers subjected to conditions mimicking ischemia followed by reperfusion (9). The changes in our studies are somewhat more pronounced and prolonged and may reflect differences in methodology (total occlusion and reperfusion in vivo versus relative hypoxia $\left(\mathrm{PO}_{2}=48\right.$ torr) in vitro) or differences in tissue type (most of the data reported in that study were from Purkinje fibers, known to be relatively resistant even to the hypoxia associated with myocardial infarction (12). In addition, superoxide radicals, free fatty acids, as well as other biochemical, mechanical, and metabolic factors inherent in an in situ, beating heart cannot be simulated exactly in in vitro ischemia and reperfusion preparations. Thus, there are numerous differences between our preparation and those of in vitro ischemia and reperfusion that may explain the differences observed. Although the techniques used are routine and standard, we cannot exclude the possibility that our dissection may have confounded our results. An incomplete return to control action potential characteristics was also demonstrated in a study by Downar et al. (8), in which continuous microelectrode recordings were made during brief ischemia followed by reperfusion in an intact porcine model demonstrating depression in action potential characteristics. Although technical factors related to making microelectrode impalements in a beating preparation were present (a depression of both action potential amplitude and resting potential were present in examples of control impalements [reference 8, Figs. 1, 3, and 5]), action potential parameters did not 
Table II. Space Constants and Time Constants of the Foot of the Action Potential in Postischemic, Reperfused Myocardium

\begin{tabular}{llll}
\hline Tissue & Conduction velocity & Space constant & $\tau_{\text {foot }}$ \\
\hline & $\mathrm{m} / \mathrm{s}$ & $\mathrm{mm}$ & $\mathrm{ms}$
\end{tabular}

Control

$\begin{array}{llll}1 & 0.56 & 1.05 & 1.28 \\ 2 & 0.53 & 0.58 & 1.01 \\ 3 & 0.45 & 0.70 & 0.81 \\ 4 & 0.64 & 0.82 & 0.80 \\ 5 & 0.81 & 0.72 & 0.89 \\ 6 & 1.75 & 1.78 & 1.43 \\ 7 & 1.22 & 1.47 & 1.31 \\ 8 & 0.70 & 1.26 & 1.46 \\ \text { Mean } \pm \text { SD } & 0.83 \pm 0.45 & 1.05 \pm 0.42 & 1.12 \pm 0.28\end{array}$

Postischemic (linear conduction)

\begin{tabular}{|c|c|c|c|}
\hline 1 & 0.34 & 0.37 & 1.57 \\
\hline 2 & 0.22 & 0.28 & 1.53 \\
\hline 3 & 0.47 & 0.57 & 1.57 \\
\hline 4 & 0.30 & 0.59 & 1.11 \\
\hline 5 & 0.35 & 0.44 & 1.00 \\
\hline 6 & 0.26 & 0.41 & 2.56 \\
\hline Mean \pm SD & $0.32 \pm 0.10^{*}$ & $0.45 \pm 0.12^{*}$ & $1.57 \pm 0.55$ \\
\hline \multicolumn{4}{|c|}{ Postischemic (nonlinear conduction) } \\
\hline 1 & 0.47 & 0.49 & nl \\
\hline 2 & 0.30 & 0.53 & 1.99 \\
\hline 3 & 0.29 & 0.41 & 3.95 \\
\hline 4 & 0.07 & 0.59 & 2.54 \\
\hline Mean \pm SD & $0.28 \pm 0.16^{*}$ & $0.51 \pm 0.08^{*}$ & $2.83 \pm 1.01^{*}$ \\
\hline $\begin{array}{c}P \text { (analysis of } \\
\text { variance) }\end{array}$ & $<0.001$ & 0.004 & 0.002 \\
\hline
\end{tabular}

nl, nonlinear.

* Significantly different from normal tissues at the 0.05 level (Newman-Keuls multiple rank test).

return to their "normal values" after reflow (reference 8, Figs. 1-3) although the follow-up time was limited. In addition, because postischemic, reperfused myocardium exhibits markedly diminished developed tension, a bias toward better impalements would be present after ischemia and reperfusion and hence any return toward normal values may have been overestimated.

The etiology of the action potential changes is unknown. A decrease in high energy phosphates has been noted in postischemic, stunned myocardium (23). This may have been a factor in altering action potential characteristics dependent, in part, on the sodium-potassium pump, such as the resting potential and the activation of the inward sodium channel. Alternatively, intracellular calcium overload, perhaps due to enhanced sodiumcalcium exchange in sodium-loaded cells, which is known to cause cell-to-cell uncoupling $(11,24,25)$ and which is present in ischemic and postischemic myocardium (26-29), may have been a contributing factor. Further support for intracellular calcium overload as a unifying hypothesis explaining the cellular electrophysiologic abnormalities identified in postischemic, reperfused myocardium is the finding that typical delayed afterdepolarizations have been seen in Purkinje fibers subjected to similar conditions (9). In addition, cell depolarization in those studies as well as the present ones is consistent with significant intracellular calcium overload. Finally, the electron microscopic studies demonstrated that the degree of ischemic injury in our model was likely close to that which leads to tissue necrosis; of the seven tissues, one tissue demonstrated early changes of reversible ischemic injury, whereas another tissue demonstrated typical changes of irreversible ischemic injury (30). Of note is that these changes were most consistent with reflow injury (contraction bands), also thought to be due to intracellular calcium overload. Although ischemic metabolites such as lactate or adenosine, as well as changes in extracellular $\mathrm{pH}$, may contribute to action potential abnormalities during acute ischemia in vivo, they are probably washed out during the reflow phase, and therefore cannot contribute to persistent electrophysiological abnormalities. In addition, because myocardium was examined in vitro in superfused preparations, the role of ischemic metabolites would be further minimized.

The present experiments demonstrate that the space constant is reduced in the ischemic zone during the recovery phase after sublethal ischemic injury. A reduced space constant was noted in each of the tissues taken from the postischemic zone (Fig. 4, Table II). A decreased space constant has previously been described in acutely hypoxic Purkinje fibers and has been attributed to an increase in internal axial resistance (18). The mechanisms responsible for this finding, in postischemic, reperfused myocardium however, are not clear. The cell diameters in the postischemic, reperfused tissues did not differ significantly from those previously measured in normal tissues and hence cannot be a factor contributing to the reduced space constant. Equation 1 shows the relationship between the space constant and internal axial, membrane, and extracellular resistances:

$\lambda^{2}=r_{m} /\left(r_{i}+r_{0}\right)$

where $\lambda$ is the space constant, $r_{m}$ is the membrane resistance, $r_{i}$ is the internal axial resistance, and $r_{0}$ is the resistance of the extracellular space (31). A decrease in the space constant could be due to a decrease in $r_{m}$ or to an increase in effective axial resistance $\left(r_{i}\right.$ and/or $\left.r_{0}\right)$. Unfortunately, we were unable to measure the input resistance in our experiments, and therefore cannot determine which of these factors contributed to the reduced space constant. Abnormalities in cell coupling due to anatomic disruption of intercellular connections are not a likely explanation, because the histologic and electron microscopic studies demonstrated that the cellular structures (including the cell nexuses) were entirely normal in five of the seven specimens even though a reduced space constant was present in all. Alternatively, as noted above, ischemia and reperfusion may lead to intracellular calcium overload (26-29), which in turn may lead to an impairment in cell coupling possibly by altering the configuration of the low resistance nexus $(24,25)$.

Other factors such as voltage-dependent active components, rectification of steady-state outward currents, and voltage-dependent steady-state inward currents may contribute to subthreshold responses. In addition, these factors may be more significant in normal tissues, as compared with depolarized postischemic, reperfused tissues, and hence may contribute to the differences in the measured space constants in the two groups. These factors tend to cause a deviation from the purely exponential relationship between voltage drop and distance of the subthreshold response. That the correlation coefficients were close to unity $(0.94 \pm 0.03$ and $0.95 \pm 0.05$ for control and post- 

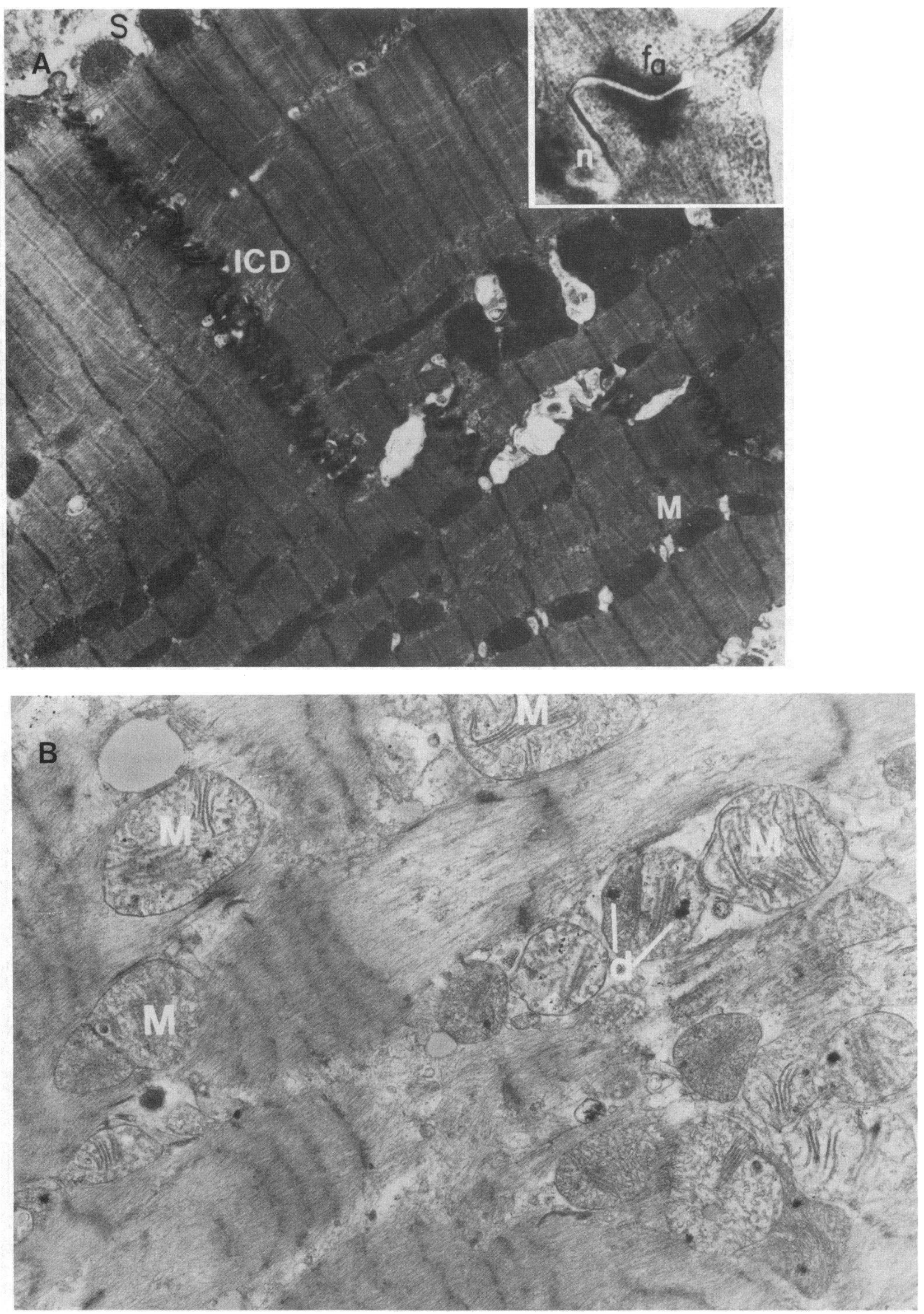


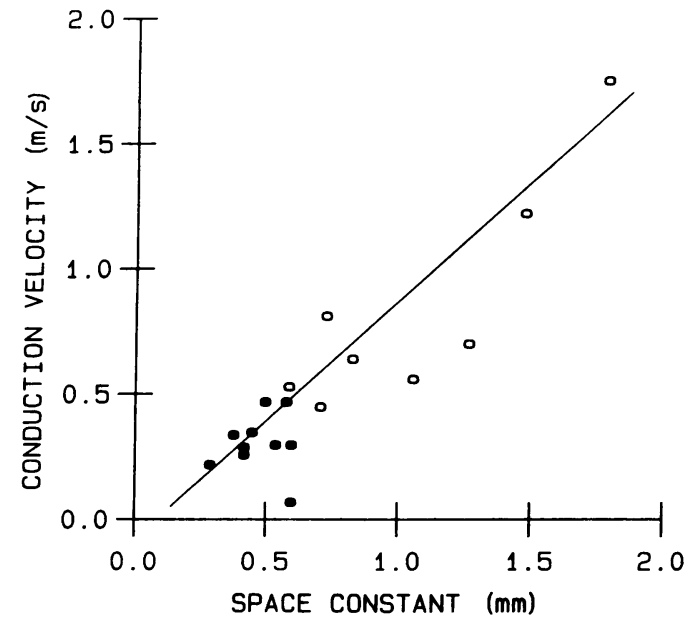

Figure 6. Relationships between conduction velocity and space constant for normal (open ovals) and postischemic, reperfused (filled ovals) tissues. The equation of the regression, correlation coefficient, and level of significance of the regression are shown for this relationship. $y=0.09+0.87 x ; R=0.09 ; P<0.001$.

ischemic tissues respectively) suggests that any error in the spaceconstant determination introduced by these factors is relatively minor. In addition, there was no difference in the correlation coefficients between control and postischemic, reperfused determinations, which indicated that there was not a systematic error in measuring the space constant in one but not the other group of tissues.

There was a wide range of conduction velocities noted in our control experiments. This was primarily due to enhanced conduction in one tissue. The reasons for this enhancement are unclear, but it appears to be accounted for by an increased space constant.

Determinants of slow conduction. To examine the determinants of conduction in this model, our results were subjected to testing using the analytical relationships expected between conduction velocity, the space constant, the foot of the action potential, and action potential characteristics (maximum rate of depolarization, action potential amplitude, etc.) if a one-dimensional cable model applies to our experimental preparations. The relationship between conduction velocity $(\theta)$ the space constant $(\lambda)$, the time constant of rise of the action potential foot $\left(\tau_{\text {foot }}\right)$, and the membrane time constant $\left(\tau_{m}\right)$ was described by Tasaki and Hagiwara (32):

$\theta=\lambda\left[\tau_{\text {foot }} \tau_{m}\right]^{1 / 2}$

In our experiments, the $57 \%$ decrease in the space constant and the $40 \%$ increase in $\tau_{\text {foot }}$ that we measured predict a $64 \%$ decrease in conduction velocity, which is very close to the $61 \%$ decrement that we measured. This indicates that a major factor in the decrement in conduction is a reduced space constant.
The statistical relationships predicted by a one-dimensional cable model between these variables was also noted. The relationship of Tasaki and Hagiwara (32) predicts a linear relationship between conduction velocity and the space constant. Similarly, the approximation of Hunter et al. (33) relates membrane and action potential properties to conduction velocity:

$\theta^{2}=\left[\mathrm{a} /\left(\mathrm{C}_{\mathrm{m}} \cdot \mathbf{R}_{\mathrm{i}}\right)\right]\left(\mathrm{Vmax} / \mathrm{V}_{\mathrm{p}}\right)$

where $\theta$ is conduction velocity, $a$ is the fiber radius, $C_{m}$ is the specific capacitance of the membrane, $R_{i}$ is the specific internal axial resistance, $V \max$ is the maximal rate of rise of the action potential upstroke, and $V_{p}$ is the action potential amplitude. This approximation predicts a linear relationship between the conduction velocity and the square root of the maximal rate of depolarization of the action potential upstroke.

Although a wide range existed for the measured space constants in both the normal and postischemic, reperfused tissues, a strong linear relationship was present between space constant and the conduction velocity in each tissue. The equation for this regression, as Fig. 6 shows, was $\theta=-0.09+0.87(\lambda) ; R$ $=0.90, P<0.001$, where $\theta$ is the conduction velocity and $\lambda$ is the space constant. A linear relationship was also found between conduction velocity and the square root of the maximum rate of depolarization. In addition, a weaker but statistically significant relationship was found between conduction velocity and the square root of the action potential amplitude. The equations of these regressions were:

maximum rate of depolarization:

$\theta=-0.24+0.08(\mathrm{Vmax})^{1 / 2} ; \quad R=0.67, \quad P<0.001$

action potential amplitude:

$\theta=-0.96+0.17\left(\mathrm{~V}_{\mathrm{p}}\right)^{1 / 2} ; \quad R=0.53, \quad P<0.02$,

where $\theta$ is the conduction velocity, $V \max$ is the maximum rate of depolarization, and $V_{p}$ is the action potential amplitude.

These findings may have relevance to the mechanism of arrhythmias seen after occlusion and reperfusion. Ventricular tachyarrhythmias that occur immediately after reperfusion (18, $22,23,25,32$ ) are likely due to the heterogeneous recovery of action potentials in the ischemic zone (8). The mechanism of arrhythmias that occur later (5-30 $\mathrm{min})$ after reperfusion is unclear. Penkoske et al. (25) and Kaplinsky et al. (18) demonstrated an accelerated idioventricular rhythm that had characteristics suggestive of automaticity. Levites et al. (22) and Murdock et al. (23), on the other hand, documented tachyarrhythmias with properties that suggested a reentrant mechanism. The data from our study provide evidence for the persistance of an electrophysiologic substrate in postischemic epicardial tissues that may support reentrant tachyarrhythmias. Automaticity and abnormal impulse initiation due to afterdepolarizations were not seen. It should be noted, however, that these mechanisms may be present in other areas of myocardium or in surviving Purkinje fibers
Figure 5. $(A)$ Electron micrograph $(6000 \times)$ showing normal-appearing myocardium typical of five of seven specimens. Sarcomeres are in register exhibiting $\mathrm{A}$ and $\mathrm{Z}$ bands, but not I bands. $M$, normal mitochondrion with tightly packed cristae; $S$, sarcolemma showing normal scalloped appearance; $I C D$, intercalated disk showing normal steplike configuration. Inset, high power $(100,000 \times)$ view of a section of the intercalated disk showing the fascia adherens $\left(f_{a}\right)$ with the typical dense filamentous net and nexuses or tight junctions $(n)$. (B) Electron micrograph $(8000 \times)$ of the only specimen that showed severe injury. Hypercontracted and stretched sarcomeres typical of contraction band necrosis are evident. Mitochondria $(M)$ are swollen and have disrupted cristae. Matrix densities $(d)$ typical of irreversible injury are present in many of the mitochondria. 
and our studies were not designed to rule in or out specific arrhythmic mechanisms.

The abnormal electrophysiologic properties that were present after occlusion and reperfusion may also contribute to the impaired mechanical function present in postischemic, stunned myocardium (16). Brief periods of ischemia without infarction lead to prolonged hemodynamic dysfunction. Because normal, synchronous contraction depends upon normal excitation-contraction coupling, abnormal cellular electrophysiology may play a role in a abnormal contraction. Tissues from several animals showed regions devoid of active responses and action potentials, which are important determinants in contraction, were abnormal in all of the tissues. In addition, the delay in onset of some action potentials and the heterogeneity in activation and repolarization may have led to inefficient, nonsynchronous contraction.

\section{Acknowledgments}

We thank Ralph Ianuzzi, William Moore, Charles Prood, and Pamela Dudeck for their technical expertise, and Christine G. Holzmueller for typing the manuscript.

This work was supported in part by grants from the National Institutes of Health (HL-28393, HL-33593, HL-07220, and HL-17655) and the W. W. Smith Charitable Trust. Dr. Levine is the recipient of a Clinician Scientist Research Award sponsored by The Johns Hopkins Medical Institutions.

\section{References}

1. Axelrod, P. J., R. L. Verrier, and B. Lown. 1975. Vulnerability to ventricular fibrillation during acute coronary arterial occlusion and release. Am. J. Cardiol. 36:776-782.

2. Kaplinsky, E., S. Ogawa, C. W. Balke, and L. S. Dreifus. 1979. Two periods of ventricular arrhythmia in the canine acute myocardial infarction model. Circulation. 60:397-403.

3. Kaplinsky, E., S. Ogawa, E. L. Michelson, and I. S. Dreifus. 1981. Instantaneous and delayed ventricular arrhythmias after reperfusion of acutely ischemic myocardium. Evidence for multiple mechanisms. Circulation. 63:333-345.

4. Levites, R., V. A. Banks, and R. H. Helfant. 1975. Electrophysiological effects of coronary occlusion and reperfusion: observations of dispersion of refractoriness and ventricular automaticity. Circulation. 52:760-765.

5. Murdock, D. K., J. M. Loeb, D. E. Euler, and W. C. Randall. 1980. Electrophysiology of coronary reperfusion arrhythmias. Circulation. 61:175-182.

6. Penkoske, P. A., B. E. Sobel, and P. B. Corr. 1978. Disparate electrophysiological alterations accompanying dysrhythmias due to coronary occlusion and reperfusion in the cat. Circulation. 58:1023-1035.

7. Tennant, R., and C. J. Wiggers. 1935. The effect of coronary occlusion on myocardial contraction. Am. J. Physiol. 112:351-361.

8. Downar, E., M. J. Janse, and D. Durrer. 1977. The effect of acute coronary artery occlusion on subepicardial transmembrane potentials in the intact porcine heart. Circulation. 56:217-224.

9. Ferrier, G. R., M. P. Moffat, and A. Lukas. 1985. Possible mechanisms of ventricular arrhythmias elicited by ischemia followed by reperfusion. Circ. Res. 56:184-194.

10. Levine, J. H., A. A. Ciuffo, L. C. Becker, M. L. Weisfeldt, and T. Guanieri. 1985. The electrophysiologic consequences of stunned myocardium. Clin. Res. 33:205A. (Abstr.)

11. DeMello, W. C. 1982. Intercellular communication in cardiac muscle. Circ. Res. 51:1-9.

12. Friedman, P. L., J. R. Steward, J. J. Fennoglio, Jr., and A. L.
Wit. 1973. Survival of subendocardial purkinje fibers after extensive myocardial infarction in dogs: in vitro and in vivo correlations. Circ. Res. 33:597-611.

13. Lazzara, R., N. El-Sherif, and B. J. Scherlag. 1974. Early and late effects of coronary artery occlusion on canine purkinje fibers. Circ. Res. 35:391-399.

14. Lazzara, R., N. El-Sherif, and B. J. Scherlag. 1973. Electrophysiological properties of canine purkinje cells in one day old myocardial infarction. Circ. Res. 33:722-734.

15. Spear, J. F., E. L. Michelson, and E. N. Moore. 1983. Cellular electrophysiologic characteristics of chronically infarcted myocardium in dogs suceptible to sustained ventricular tachyarrhythmias. J. Am. Coll. Cardiol. 1:1099-1110.

16. Bonke, F. I. M. 1973. Passive electrical properties of atrial fibers of the rabbit heart. Pflugers Arch. Eur. J. Physiol. 339:1-15.

17. Spear, J. F., E. L. Michelson, and E. N. Moore. 1983. Reduced space constant in slowly conducting regions of chronically infarcted canine myocardium. Circ. Res. 53:176-185.

18. Wotczak, J. 1979. Contractures and increase in internal longitudinal resistance of cow ventricular muscle induced by hypoxia. Circ. Res. 44:88-95.

19. Spach, M. S., W. I. Miller, III, D. B. Geselowitz, R. D. Barr, M. N. Kootsey, and E. A. Johnson. 1981. The discontinuous nature of propagation in normal canine cardiac muscle. Circ. Res. 48:39-54.

20. Woodbury, J. W., and W. E. Crill. 1961. On the problem of impulse conduction in the atrium. In Nervous Inhibition. E. Florey, editor. Pergamon Press, Inc., Elmsford, NY. 124-135.

21. Clerc, L. 1976. Directional differences of impulse spread in trabecular muscle from mammalian heart. J. Physiol. (Lond.) 255:335346.

22. Sano, T., N. Takayama, and T. Shimamoto. 1959. Directional difference of conduction velocity in the cardiac ventricular syncytrium studied by microelectrode. Circ. Res. 7:262-267.

23. Braunwald, E., and R. A. Kloner. 1982. The stunned myocardium: prolonged, post-ischemic ventricular dysfunction. Circulation. 66:11461150.

24. DeMello, W. C. 1975. Effect of intracellular injection of calcium and strontium on cell communication in heart. J. Physiol. (Lond.). 250: 231-245.

25. DeMello, W. C. 1976. Influence of the sodium pump on intercellular communication in heart fibres: effect of intracellular injection of sodium ion on electrical coupling. J. Physiol. (Lond.). 263:171-197.

26. Renlund, D. G., G. Gerstenblith, E. G. Lakatta, W. E. Jacobus, C. E. Kallman, and M. L. Weisfeldt. 1984. Perfusate sodium during ischemia modifies post-ischemic functional and metabolic recovery in the rabbit heart. J. Mol. Cell. Cardiol. 16:795-801.

27. Naylor, W. G., S. E. Perry, J. S. Elz, and M. J. Daly. 1984. Calcium, sodium and the calcium paradox. Circ. Res. 55:227-237.

28. Zimmerman, A. N. E., and W. C. Hulsman. 1966. Paradoxical influence of calcium ions on the permeability of the cell membrane in isolated rat hearts. Nature (Lond.). 211:646-647.

29. Ganote, C. E., and W. G. Nayler. 1985. Contracture and the calcium paradox. J. Mol. Cell. Cardiol. 17:733-745.

30. Jennings, R. B., J. Schaper, M. L. Hill, C. Steenbergen, and K. A. Reimer. 1985. Effect of reperfusion late in the phase of reversible ischemic injury. Circ. Res. 56:262-278.

31. Jack, J. J. B., D. Noble, and R. W. Tsien. 1975. In Electric Current Flow in Excitable Cells. Clarendon Press, Oxford, England. 83-97, 98130.

32. Tasaki, I., and S. Hagiwara. 1957. Capacity of muscle-fiber membrane. Am. J. Physiol. 188:324-429.

33. Hunter, P. J., P. A. McNaughton, and D. Noble. 1975. Analytical models of propagation in excitable cells. Prog. Biophys. Mol. Biol. 30: 99-144. 